\title{
Lithium abundances in Bulge-like SMR stars
}

\author{
Beatriz Barbuy $^{1}$, M. Trevisan ${ }^{1}$, B. Gustafsson ${ }^{2}$, K. Eriksson ${ }^{2}$, \\ M. Grenon ${ }^{3}$, and L. Pompéia ${ }^{4}$ \\ ${ }^{1}$ Universidade de São Paulo, Brazil \\ email: barbuy@astro.iag.usp.br \\ ${ }^{2}$ Uppsala Universitet, Sweden \\ ${ }^{3}$ Observatoire de Genève, Switzerland \\ ${ }^{4}$ Universidade do Vale do Paraíba, Brazil
}

\begin{abstract}
We analyze a sample of 21 super-metal-rich (SMR) stars, using high-resolution échelle spectra obtained with the FEROS Spectrograph at the $1.5 \mathrm{~m}$ ESO telescope. The metallicities are in the range $0.15<[\mathrm{Fe} / \mathrm{H}]<0.5,3$ of them in common with Pompéia et al. (2002). Geneva photometry, astrometric data from Hipparcos, and radial velocities from CORAVEL are available for these stars. The peculiar kinematics suggests the thin disk close to the bulge as the probable birthplace of these stars (Grenon 1999). From Hipparcos data, it appears that the turnoff of this population indicates an age of 10-11 Gyr (Grenon 1999). Detailed analysis of the sample stars is carried out. Lithium abundances of these stars were derived, and their behaviour with effective temperature is shown.
\end{abstract}

Keywords. Stars: abundances, formation - Galaxy: formation

\section{Introduction}

Grenon $(1989,1999)$ selected a sample of about 6000 dwarf stars from the NLTT catalogue (see Grenon 1999), and studied them by means of Geneva photometry, radial velocity and Hipparcos astrometry. A sub-sample of super-metal-rich (SMR) stars was revealed (Grenon 1972, 1989, 1990, 1998, 2000). Optical spectra were obtained for 21 among the most metal-rich dwarfs of the sample, with the FEROS Spectrograph at the $1.52 \mathrm{~m}$ telescope at ESO, La Silla. The total wavelength coverage is $3560-9200 \AA$ with a resolving power $\mathrm{R}=48,000$.

The surface gravities $\log g$ were derived using Hipparcos parallaxes $\pi$ with bolometric correction relations given in Alonso et al. (1995). The parameters $\left[\mathrm{T}_{\mathrm{eff}}, \mathrm{v}_{\mathrm{t}}\right]$ were obtained by fixing trigonometric surface gravities and imposing excitation equilibrium for $\mathrm{Fe} \mathrm{I}$ lines and ionization equilibrium for $\mathrm{Fe} I$ and $\mathrm{Fe}$ II. Independence between equivalent widths and the abundances of $\mathrm{Fe} I$ lines was imposed to determine $\mathrm{v}_{\mathrm{t}}$. Derivations of metallicity were carried out through the Meudon code ABON2, and the code by the Uppsala group BSYN/EQWI. We are going through iterative checks, by computing FeI and FeII in the Sun, with both codes, and then for the stars, using the same tailored MARCS models. The codes are found to generate similar results, although some minor differences, which are not expected to affect the present results, are still being explored. The comparison, and more abundance results will be presented by Trevisan et al. (2009, in prep.). Lithium abundances were derived from the LiI $6707.8 \AA$ line for the sample stars, by fitting the synthetic to the observed spectra. The spectrum synthesis code is described in Cayrel et al. (1991) and Barbuy et al. (2003). Photospheric 1D models for the sample were extracted from the MARCS grid (Gustafsson et al. 2008). 


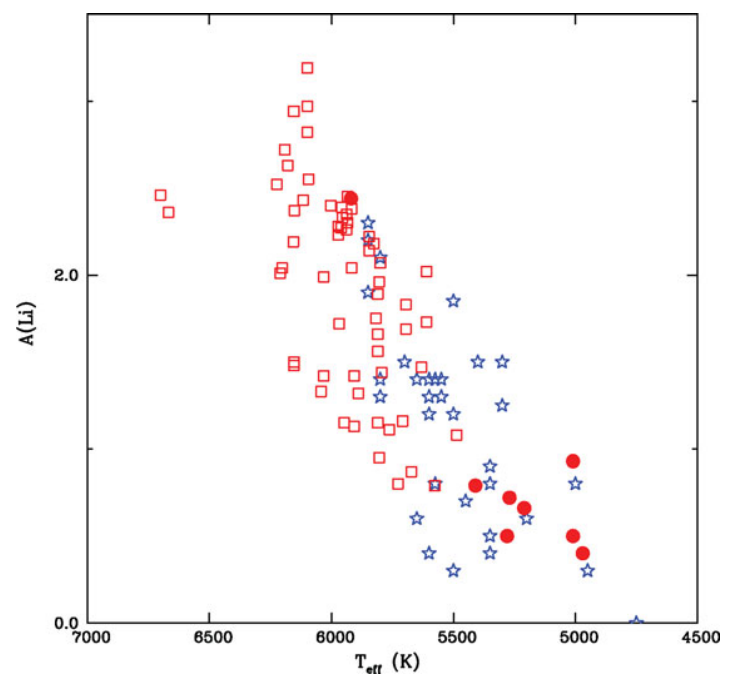

Figure 1. $\log (\mathrm{Li} / \mathrm{H})$ vs. effective temperature for the sample stars (red filled circles), compared with data for another sub-sample of bulgelike SMR stars by Pompéia et al. (2002) (open stars), and the metal-rich open cluster M67 (open squares, with results from Jones et al. (1999).

Comparisons are presented in Fig. 1 with the results by Pompéia et al. (2002), obtained for another sub-sample of bulgelike dwarfs, therefore compatible in terms of age and kinematics with the present sample, and with M67, a metal-rich open cluster of 4.5 Gyr, with data from Jones et al. (1999). Jones et al. had also shown that older stars deplete their $\mathrm{Li}$ with time, and it is more efficient in the cooler stars.

In conclusion, lithium abundances in our sample of very metal-rich stars are somewhat higher than in M67, and those found by Pompéia et al. (2002) for similar stars. One star HD 90054 shows a high Li abundance, compatible with being the hottest star of the sample.

\section{Acknowledgements}

The observations were carried out within brazilian time in a ESO-ON agreement, and within an IAG-ON agreement funded by FAPESP project $n^{\circ} 1998 / 10138-8$.

\section{References}

Alonso, A., Arribas, S., \& Martinez-Roger, C. 1995, A\&A, 297, 197

Barbuy, B., Perrin, M.-N., Katz, D. et al. 2003, A\&A, 404, 661.

Cayrel, R., Perrin, M.-N., Barbuy, B., \& Buser, R. 1991, A\&A A, 247, 108.

Grenon, M. 1972, in IAU Colloq. 17, Age des Etoiles, eds. G. Cayrel de Strobel \& A. M. Deplace (Paris : Obs. de Paris-Meudon), 55.

Grenon, M. 1989, Ap\&SS, 156, 29.

Grenon, M. 1990, in ESO Workshop and Conf. Proc. 35, Bulges of Galaxies,1 st ESO / CTIO

Workshop, ed. B. Jarvis \& D. Terndrup (Garching : ESO), (Sunspot : NSO), 143.

Grenon, M. 1998,Highlights of Astronomy, Vol.11A, ed. J. Andersen (Dordrecht:Kluwer), 560.

Grenon, M. 1999, ApESSS, 265, 331.

Grenon, M. 2000, in The Evolution of the Milky Way, ed. F. Matteucci \& F. Giovannelli (Dordrecht : Kluwer), 47.

Gustafsson, B., Edvardsson, B., Eriksson, K. et al. 2008, A\& A, 486, 951

Jones, B. F., Fischer, D., \& Soderblom, D. R. 1999, AJ, 117, 330

Pompéia, L., Barbuy, B., Grenon, M., \& Castilho, B. V. 2002, ApJ, 570, 820 